\title{
INVERSE METHOD FOR THE RETRIEVAL OF OCEAN VERTICAL PROFILES USING SELF ORGANIZING MAPS AND HIDDEN MARKOV MODELS. Application on ocean colour satellite image inversion.
}

\author{
Charantonis Anastase Alexandre ${ }^{1}$, Brajard Julien ${ }^{1}$, Moulin Cyril ${ }^{2}$, Bardan Fouad $^{3}$, and Thiria Sylvie ${ }^{1}$ \\ ${ }^{1}$ Laboratoire d'Océanographie Climat et Analyses Numériques, Université Pierre et Marrie Curie, Tour 45-55, 4, Place \\ Jussieu, 75252, Paris, France \\ ${ }^{2}$ Laboratoire des Sciences du Climat et de l'Environnement, L'Orme des Merisiers, CEA Saclay, bat 712, 91191, Gif-sur- \\ Yvette, France \\ ${ }^{3}$ Laboratoire CEDRIC,Conservatoir National des Arts et Metiers (CNAM), 292, rue Saint Martin, 75003, Paris, France \\ \{Anastase-Alexandre.Charantonis, Julien.Brajard,Sylvie.Thiria\}@locean-ipsl.upmc.fr, \\ cyril.moulin@lsce.ipsl.fr, \\ fouad.badran@cnam.fr
}

\begin{abstract}
Keywords: $\quad$ Self Organising Maps; Hidden Markov Models; Inversion; Geophysical; Chlorophyll-A; Satellite imaging; Inversion.

Abstract: $\quad$ This paper presents a statistical inversion method used to infer 3D data from 2D imaging. The methodology is based on a combination of the Self Organising Maps and the Hidden Markov Models. The SelfOrganising Maps generate the typical situations of the emissions and the hidden states of the Hidden Markov Model. The method has been validated by inferring the oceanic vertical profiles of Chlorophyll-A based on sea-surface data.
\end{abstract}

\section{INTRODUCTION}

The density of satellite observations allowed a semi-continuous observation of the global ocean surface. The two-dimensional images provided by this coverage often contain information on integrated quantities whose vertical distribution is unknown. Depending on the field of study there exist different dynamic approaches for inverting this type of data. However, these approaches are often faced with the problem of non-linearity, and can also be hampered by a lack of knowledge of the complete mechanisms that govern the distributions.

The present paper deals with the inversion of observed sea-surface satellite images, noted $\tilde{x}_{o b s}^{t}$ $t \in[1 \cdots T]$, for retrieving of the vertical distribution of Chlorophyll-A, noted $\tilde{x}_{d i s}^{t} t \in[1 \cdots T]$, using a statistical, non-linear approach.

The methodology we have developed is a mixture of the neuronal algorithms known as Self Organizing Topological Maps (SOM) and the Hidden Markov Models (HMM). The SOM are unsupervised classification algorithms, that allow us to cluster our availiable data into classes. The classes are arranged on a topological map and connected to each other by a topological similarity distance. In the present study the SOM classification is applied twice, once on the sea-surface data, and once upon the vertical profiles connected to these images. The resulting topological maps allow us to discretize both data spaces into set amounts of classes.

The second statistical algorithm, the HMM allows us to infer the most likely sequence of some discrete, unobservable states, given a series of discrete, observable states. To do so a set of probability matrices are calculated, corresponding to the dynamic processes of the unobserved states and the links existing between the observed and unobserved states. We use the classes created through the SOMs to discretize the availiable data and therefore represent both the observable and the unobservable states.

In this paper, we present the results obtained with the methodology developed on a case study at the site of the Bermuda Atlantic Time Series (BATS) (32 N -64 W) of the JGOFS campaign. 

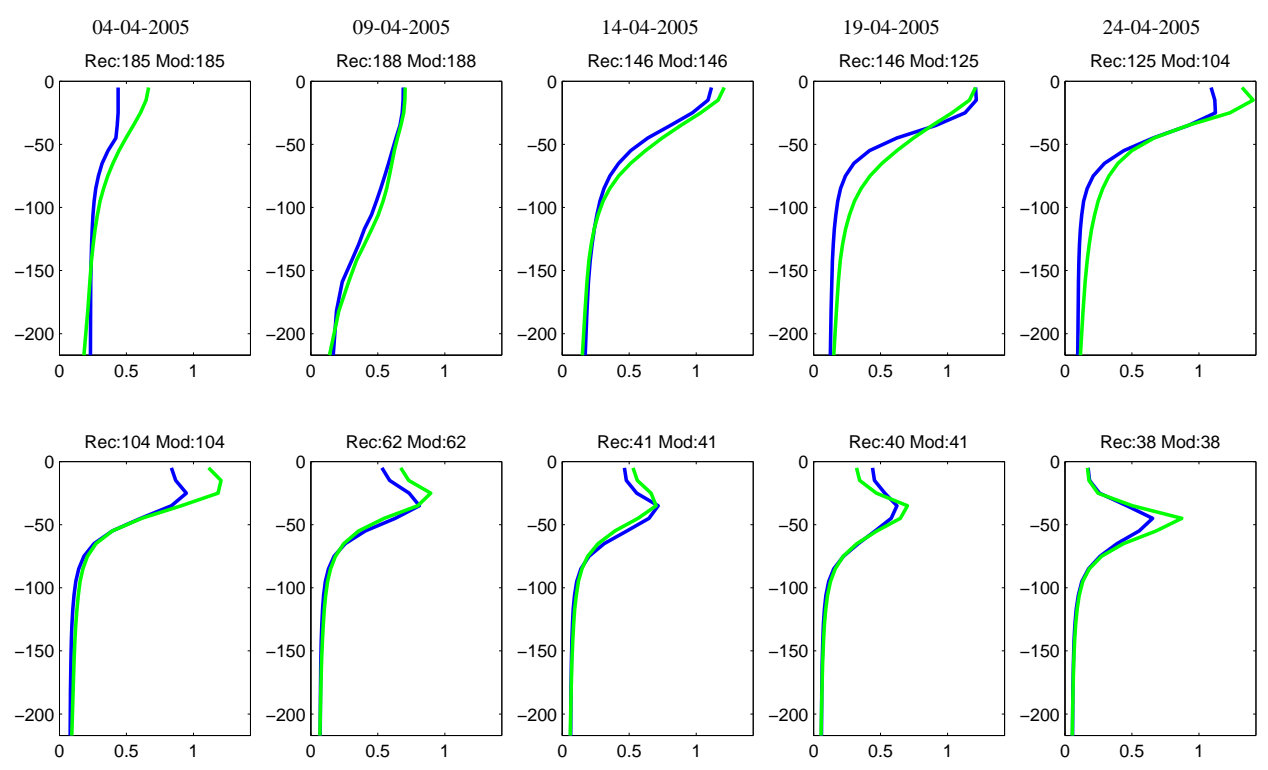

29-04-2005

04-05-2005

09-05-2005

14-05-2005

19-05-2005

Figure 1: Inversion of ten 5-days steps for the period from 04-04-2005 to 05-19-2005, at BATS. In green, the states provided by the inverse method and in blue the vertical distribution of Chlorophyll-A according to the NEMO-PISCES model. The horizontal axes are in $10^{6} \mu \mathrm{mol} / \mathrm{L}$ of Chlorophyll-A, while the vertical ones are in meters from the sea surface. The numbers on top correspond, after $\boldsymbol{R e c}$, to the indexes of the classes on $\mathrm{M}_{\mathrm{dis}}$ attributed to that 5-day step by the inverse method, and, after $\boldsymbol{M o}$ to the indexes attributed by projection of the total profile on $\mathrm{M}_{\text {dis }}$. These are not show in the figure.

\section{SELF-ORGANIZING TOPOLOGICAL MAPS}

Self-Organising Topological Maps (SOM) are clustering methods based on neural networks $(\mathrm{S}$, Haykin 1999). They provide a discretization of a learning dataset $\mathrm{A}=\left\{\tilde{x}_{k} \in R^{p}, \mathrm{k}=1 \ldots \mathrm{N}\right\}$ into a reduced number of subsets, called classes, $\mathrm{P}_{\mathrm{i}},\{\mathrm{i}=$ 1...M that share some common statistical characteristics. Each subset is represented by its referent vector $\boldsymbol{r}_{i}$ which approaches the mean value of the elements in the class $\mathrm{Pi}$. In our case, we trained two SOMs, one containing the observations, called $\mathrm{M}_{\mathrm{obs}}$ and one containing the distributions of the unobservable states, called $\mathbf{M}_{\text {dis }}$. The number of classes in $\mathrm{M}_{\mathrm{obs}}$ and $\mathrm{M}_{\text {dis }}$ are respectively noted $\mathrm{N}_{\mathrm{obs}}$ and $\mathrm{N}_{\text {dis }}$.

The topological aspect of the maps can be justified if we consider the Map as an undirected graph on a two-dimensional lattice whose vertices are the $\mathrm{m}$ classes. This graph structure therefore allows the definition of an discrete distance $d(i, j)$ between two classes $i$ and $j$, defined as the length of the shortest path between $i$ and $j$ on the map. The nature of the SOM training algorithm forces a topological ordering upon the map, and therefore any neighbouring classes $\boldsymbol{c}_{\boldsymbol{i}}$ and $\boldsymbol{c}_{j}$ on the map have referent vectors $\boldsymbol{r}_{i}$ and $\boldsymbol{r}_{j}$ that are close in the Euclidian sense in the data space $\mathrm{R}^{\mathrm{P}}$. The topological ordering constitutes a major element of our inverse method, since it allows us to make, latter on, the ergodic assumption for our Markov states.

We define a series of observable events by taking the data from observations related to a given period of time and we label each observation by the index of the class to which it is assigned by using $\mathrm{M}_{\mathrm{obs}}$. This classification is done by allocating to each observation $\tilde{x}_{o b s}^{t} t \in[1 \cdots T]$, in the sequence the index of the class of $M_{\text {obs }}$ whose referent is the closest to it in the Euclidian sense. Therefore we obtain the series

$$
\begin{gathered}
\mathrm{S}_{\mathrm{obs}}=\left\{s_{o b s}^{t}=\operatorname{argmin}_{i}\left(\left|\tilde{x}_{o b s}^{t}-\boldsymbol{r}_{i}^{o b s}\right|\right),\right. \\
\left.i=1 \cdots N_{o b s}\right\}
\end{gathered}
$$

In the same way, we obtain the time-series of distributions

$$
\mathrm{S}_{\mathrm{dis}}=\left\{s_{d i s}^{t}=\underset{\operatorname{argmin}_{i}\left(\left|\tilde{x}_{d i s}^{t}-\boldsymbol{r}_{i}^{d i s}\right|\right), i=}{\left.1 \cdots N_{\text {dis }}\right\}}\right.
$$



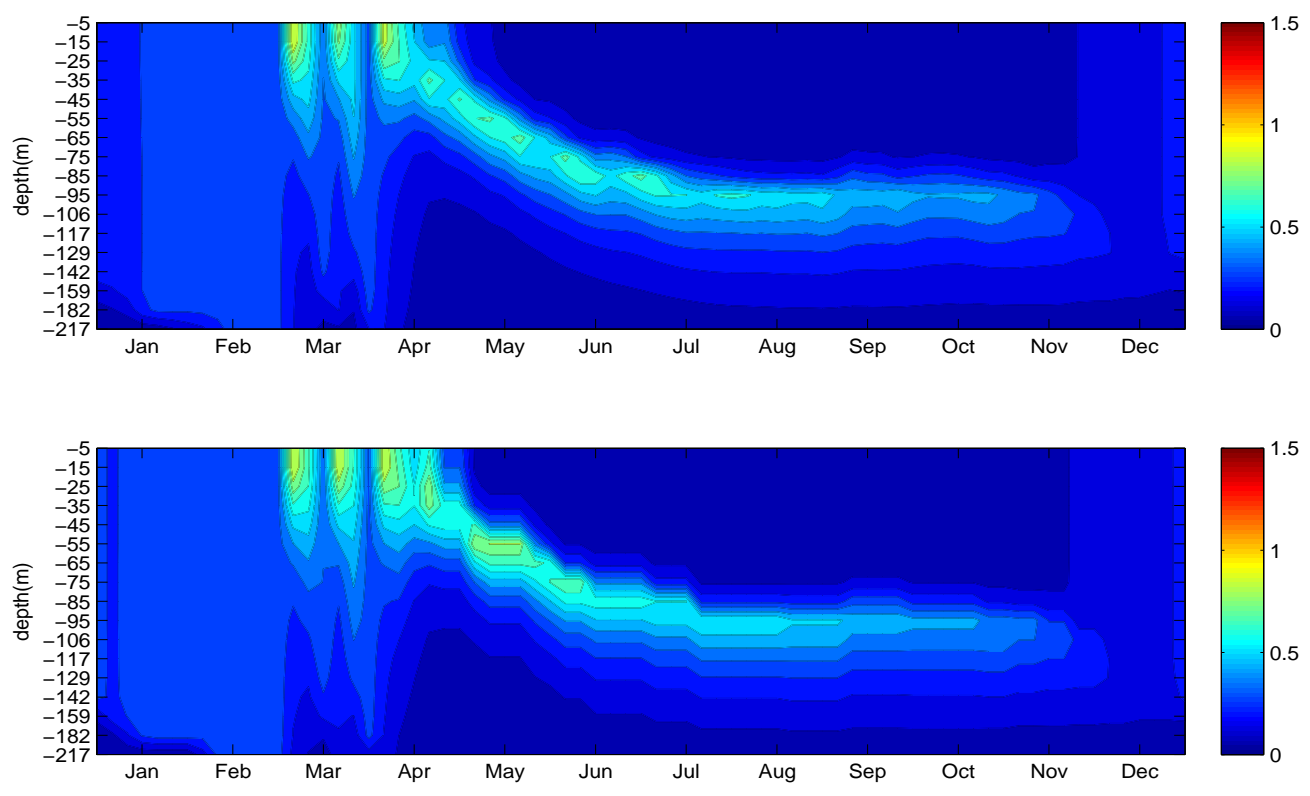

Figure 2: The reconstruction at BATS of the validation year 2005, according to, the NEMO-PISCES MODEL (top graph) and the inverse method result (bottom graph). The colorbar indicates the Chlorophyll-A concentration in $10^{6}$ $\mu \mathrm{mol} / \mathrm{L}$.

These series $S_{\text {obs }}$ and $S_{\text {dis }}$ are used by the HMM in order to estimate the probabilistic links that exist between the observable states and the unobservable ones.

The referents $\boldsymbol{r}_{\mathrm{dis}}$ of $\mathrm{M}_{\mathrm{dis}}$ are also used as the vertical profiles in our sequence reconstructions.

\section{HIDDEN MARKOV MODELS}

A Markov model is a stochastic model that assumes the first order Markovian property, meaning that each consecutive state of the model depends solely on its previous stat of the model such as

$$
\mathrm{P}\left(\mathrm{X}_{\mathrm{t}} \mid \mathrm{X}_{1} \mathrm{X}_{2} \ldots \mathrm{X}_{\mathrm{t}-1}\right)=\mathrm{P}\left(\mathrm{X}_{\mathrm{t}} \mid \mathrm{X}_{\mathrm{t}-1}\right)
$$

Expanding this principle, a Hidden Markov Model (HMM) is a stochastic model with two sequences. One sequence of unobservable states that follow the first order Markovian property, (represented in our method by $S_{\mathrm{dis}}$ ), and one sequence of observable states, (represented by $\mathrm{S}_{\mathrm{obs}}$ ), that have a statistical link with the unobservable states (O. Cappé et al. 2005).

We consider two phases, a training one, and a retrieval one. During the training, the Transitions matrix $\operatorname{Tr}$ and the Emissions matrix Em are estimated. Tr contains the transition probabilities of the unobserved states

$$
\operatorname{tr}_{\mathrm{i}, \mathrm{j}}=\mathrm{P}\left(\mathrm{C}_{\mathrm{dis}(\mathrm{i}, \mathrm{t})} \mid \mathrm{C}_{\mathrm{dis}(\mathrm{j}, \mathrm{t}-1)}\right)
$$

where

$$
\sum_{i=1}^{N_{d i s}} t_{i, j}=1
$$

$\mathrm{Tr}$ corresponds, in a physical sense, to the underlying dynamics that govern the unobserved states.

Em contains the à posteriori probabilities of each observed state to have been emitted by an unobserved state,

$$
\mathrm{e}_{\mathrm{i}, \mathrm{j}}=\mathrm{P}\left(\mathrm{C}_{\mathrm{dis}(\mathrm{i}, \mathrm{t})} \mid \mathrm{C}_{\mathrm{obs}(\mathrm{j}, \mathrm{t})}\right)
$$

where

$$
\sum_{j=1}^{N_{d i s}} e_{i, j}=1
$$

Em corresponds, in a physical sense, to the link existing between the observed quantities and the dynamics of the unobserved quantities. Another probability matrix that needs to be calculated is the initial probability matrix $\Pi$, with components $\pi_{\mathrm{i}}$ which represent the average revisit rate of each 

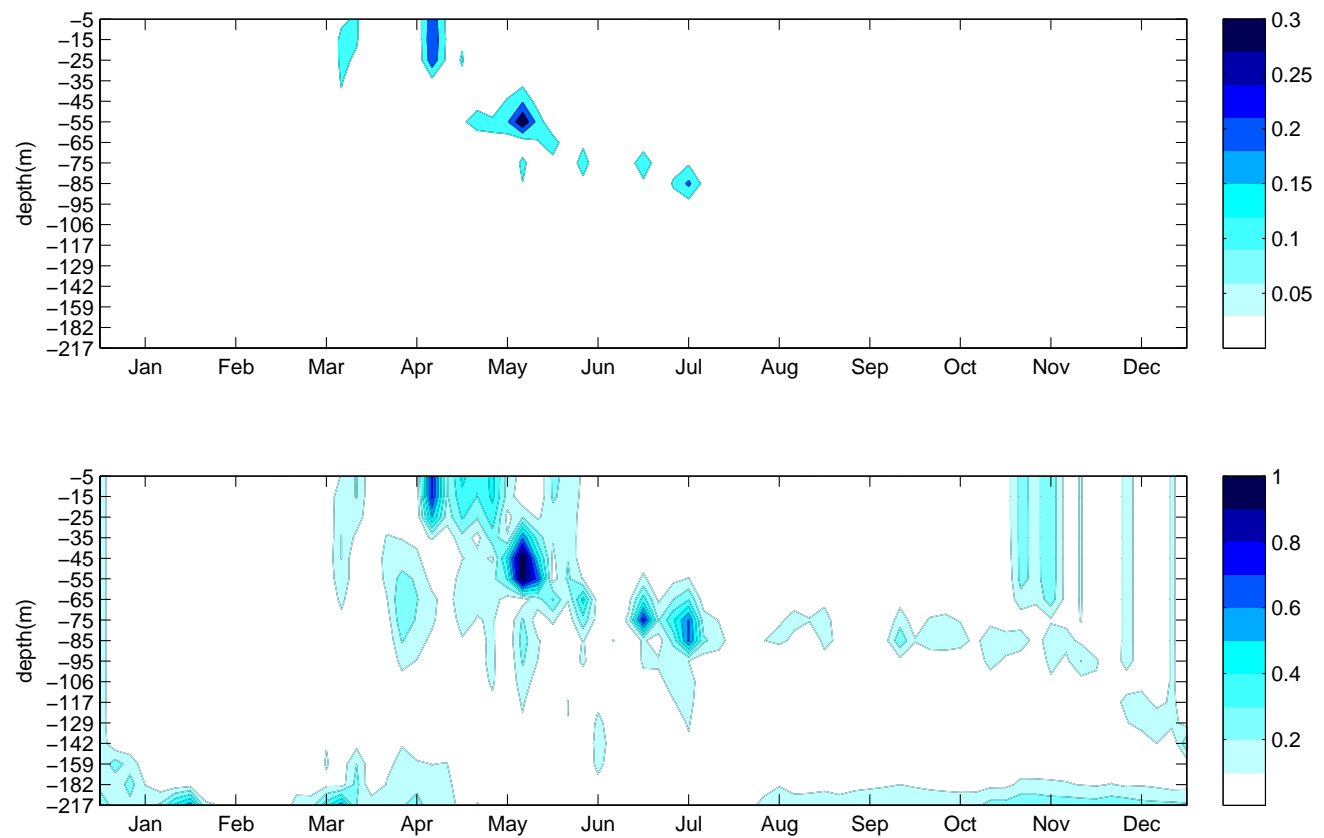

Figure 3: The top image contains the absolute error between the NEMO-PISCES model and the result of the inverse method, at BATS for the validation year 2005. The bottom image contains the absolute relative error between the NEMO-PISCES model and the result of the inverse method.

unobserved state given an infinite sequence. All mentioned probabilities are estimated by using the Baum-Weltch algorithm (L. E. Baum et al. 1970), which is a maximum likelihood optimization algorithm, that takes as input the sequences $S_{\text {obs }}$ and $\mathrm{S}_{\mathrm{dis}}$ and outputs the most likely matrices to have generated them through a hidden Markov process.

During the recognition phase, we used the Viterbi algorithm, which is a well-know dynamic programming algorithm (Viterbi AJ, 1967), for inferring the most likely sequence of indexes $\mathrm{S}_{\mathrm{dis} \text {-est }}$ representing the unobserved states, given the previously estimated parameters $\mathrm{Tr}, \mathrm{Em}$ and $\Pi$ of the HMM and a sequence of observations $S_{\text {obs-new. It is }}$ well documented (M.S. Ryan and G.R. Nudd. 1993) that the Viterbi algorithm can face problems due to transitions that were not observed in the training data set. A balance needs to be found between the sizes of the SOM maps that will determine the amount of discretization provided by the method, and the correctness of the allocation of indices. The dimensions of each map are therefore optimized using a validation set. Yet, even with an optimization there will be some situations and transitions that are seldomly encountered in the training data and result in null probabilities in the probability matrices $\mathrm{Em}_{\mathrm{B}-\mathrm{W}}$ and $\mathrm{Tr}_{\mathrm{B}-\mathrm{W}}$ that we estimated in the first pass of the Baum-Weltch algorithm.
Due to this usual lack of sufficient data in the concerned domains, $\mathrm{Em}_{\mathrm{B}-\mathrm{W}}$ and $\mathrm{Tr}_{\mathrm{B}-\mathrm{W}}$ need to be adjusted. This is done by taking into account the properties of the topological maps. A major characteristic of the present method is to use the topological order in order to improve the accuracy of the estimated probabilities matrices. The topological maps allow us to modify the probabilities by allowing each state to communicate via a diminutive probability with each of its neighbouring states.

This is done by considering the neighbourhood matrices $\mathrm{NM}_{\mathrm{obs}}$ and $\mathrm{NM}_{\mathrm{dis}}$, of dimensions $\left(\mathrm{N}_{\mathrm{obs}}, \mathrm{N}_{\mathrm{obs}}\right)$ and $\left(\mathrm{N}_{\text {dis }}, \mathrm{N}_{\text {dis }}\right)$, where

$$
\mathrm{NM}_{\mathrm{SOM}}(\mathrm{i}, \mathrm{j})=\left\{\begin{array}{c}
1, \text { if } \mathrm{d}\left(c_{i}^{\text {SOM }}, c_{j}^{\text {SOM }}\right)<2 \\
0, \text { else }
\end{array}\right.
$$

with $d(i, j)$ being the discrete distance of the map. Taking into account the neighbourhood consists in increasing the probability of reaching a class $j$ from a class $i$, by an ammount proportional to the sum of the previously calculated probabilities of reaching the neighbour classes of class j on SOM. In order to favour the data observed during training, we add a weighting term, noted $\mathrm{w}_{\mathrm{c}}$, to the initial probabilities, and we further multiply it by the total length of the training sequences used in the intitial Baum-Weltch algorithm's pass, noted $\mathrm{T}_{\text {training, }}$, since this length is a 
measure of confidence in the correctness of the estimated parameters. The matrices obtained are then normalized. The final Em and Tr matrices we use, noted $\mathrm{Em}_{\text {final }}$ and $\mathrm{Tr}_{\text {final }}$, are computed by applying:

$$
\begin{gathered}
\operatorname{Em}_{\text {final }}(i, j)=w_{c} * T_{\text {training }} * \\
\operatorname{Em}_{B-W}(i, j)+\sum_{k=1}^{N_{o b s}}\left(N M_{\text {obs }}(i, k) *\right. \\
\operatorname{Em}(i, k))+1
\end{gathered}
$$

Which is normalized to fit the constraint (7), and

$$
\begin{gathered}
\operatorname{Tr}_{\text {final }}(i, j)=w_{c} * T_{\text {training }} * \\
\operatorname{Tr}_{B-W}(i, j)+\sum_{k=1}^{N_{\text {dis }}}\left(N M_{\text {dis }}(i, k) *\right. \\
\operatorname{Em}(i, k))
\end{gathered}
$$

Which is normalized to fit the constraint (5). For this application $\mathrm{w}_{\mathrm{c}}$ is set to 9 .

These modifications permit the Viterbi algorithm to circumvent the problems of impossible transitions, or emissions due to insufficient data in the training sequences that resulted in nul probabilities in the estimated parameters.

\section{APPLICATION FOR THE RESTITUTION OF THE VERTICAL CHLOROPHYLL-A CONCENTRATION THROUGH SEA SURFACE DATA}

The bio-geochemical activity of the oceans and the carbon cycle are two parts of a complex feedback system. A change in climate and an increase of the amount of available carbon can affect the primary oceanic production, and in return a change in the bio-geochemical activity affects, by modifying the albedo and carbon fixation rates, the climate and carbon concentration. It is therefore important to be able to determine the oceanic primary production. In recent years, many algorithms have been developed that infer the Chlorophyll-A concentration in ocean surface layers through satellite imaging (Brajard et al. 2008). It has also been proved that the vertical Chlorophyll-A distribution, is correlated with sea surface data (Uitz et al. 2006). Therefore, the determination of the vertical distribution of Chlorophyll-A from sea surface data is a problem that can be solved by the methodology we propose.

One cannot determine the vertical distribution of Chlorophyll-A without first understanding the parameters that influence the development of phytoplankton. It is generally accepted that phytoplankton growth depends on 5 parameters: available radiation, available nutriments, predators and biology, water temperature, water turbidity.

These parameters cannot easily be monitored through a direct approach. Satellite imaging, however, can give us proxy information, which can be used in an empirical approach for determining the vertical distribution of Chlorophyll-A. Specifically, in this study we used: Sea Surface Chlorophyll-A concentration (SCHL), Sea Surface Temperature (SST), Sea Surface Elevation (SSH), Shortwave Radiation (SR) and Wind-speed Intensity (WS).

Since our objective is to validate the theoretical methodology, we used simulated data in order to test the validity of our approach. We therefore approximated the satellite values of the previous parameters by using the input and output values provided by the NEMO oceanic circulation model coupled to the PISCES bio-geochemical model (C. Moulin, 2008). In order to better simulate the noise and errors inherent to satellite images we added a white noise $\mathrm{z} \sim \mathrm{N}(0, \hat{\mathrm{e}}), \hat{\mathrm{e}}=1 / 2 *\left(\sigma_{\mathrm{schl}}, \sigma_{\mathrm{sst}}, \sigma_{\mathrm{ssh}}, \sigma_{\mathrm{ws}}\right.$, $\left.\sigma_{\mathrm{sr}}\right)$ to the parameters that could be gathered from satellite imaging. $\sigma$ represents the standard derivation of each corresponding surface parameter, as computed on the training data. The application was set at the site of the Bermuda Atlantic Time Series (BATS) (32 N $-64 \mathrm{~W})$ of the JGOFS campaign.

The unobserved states that were classified, were the output data vectors containing the average vertical Chlorophyll-A distribution at 17 depth levels (from 5 meters to 217) and temperature distribution at 9 depth levels. These vertical distribution profiles were 5-day averages spanning the period from 1991 to 2007 located in a $2^{\circ} \times 2^{\circ}$ square centred on BATS. Therefore $\mathrm{M}_{\text {dis }}$ belongs to $\mathrm{R}^{26}$ (17 levels of Chlorophyll-A +9 levels of Temperature). $\mathrm{M}_{\mathrm{obs}}$ belongs to $\mathrm{R}^{5}$.

We trained $M_{\text {obs }}$ and $M_{\text {dis }}$ by taking into account all available profiles at BATS, as well as any adjacent points included in the model. This gave us $9 * 73 * 17=11169$ profiles for the construction of the maps. The optimum map sizes, $\mathrm{N}_{\mathrm{obs}}$ and $\mathrm{N}_{\mathrm{dis}}$ were determined to be $21 * 14=294$ classes.

For the estimation of the HHM parameters on the other hand, we take a total of 14 years (19912004) for the training, each including seventy-three 5-day steps. Therefore $\mathrm{T}_{\text {training }}=1022$. We maintained 3 years (2005-2007), or 219 5-day steps, to validate our approach.

The results shown in Figure 1 present the temporary evolution of Chlorophyll-A profiles in ten 5-day steps sequence, from 04-04-2005 to 19-052005, at BATS. In green we see the Chlorophyll-A distribution profiles, taken from the referents $\boldsymbol{r}_{d i s}$ of 
$\mathrm{M}_{\mathrm{dis}}$, corresponding to the indexes of the reconstructed time series $S_{\text {dis-rec }}$. In blue we can see the vertical distribution of Chlorophyll-A according to the NEMO-PISCES model at the same 5-day steps.

In Figure 1 we also have, preceded by the acronym $\boldsymbol{R e c}$, the indexes that constitute time series $\mathrm{S}_{\text {dis-rec }}$ and, preceeded by the acronym Mod, the indexes we obtain by projecting the corresponding profiles of the NEMO-PISCES model on $\mathrm{M}_{\mathrm{dis}}$. In order to avoid confusion, the profiles corresponding to the indexes after Mod are not displayed. When the vertical distribution of Chlorophyll-A is know, these indexes would correspond to the optimum reconstruction we could get with $\mathrm{M}_{\text {dis. }}$. We note this optimum time series as $\mathrm{S}_{\text {dis-opt. }}$ It is interesting to notice that even when the indexes are not equal, the classes are neighbours on $\mathbf{M}_{\mathrm{dis}}$, and the estimated profiles are quite similar to the observed ones.

If we define $S_{\text {dis-2005to2007 }}$ as the reconstructed time series of indexes of the validation years from 2005 to 2007 and as $S_{\text {dis-opt-2005to2007 the }}$ corresponding optimum reconstruction we observe that they are in agreement $84,59 \%$ of the time. This perforance reaches $88,58 \%$ when applied on the reconstruction $S_{\text {dis-2005 }}$ of the year 2005 alone, as compared to its optimum reconstruction. This was probably due to the validation year 2005 having a small variation from the mean year, and presenting often-observed transitions. In the training date we had on average an agreement of $86,46 \%$

In Figures 2 and 3, we applied the inverse method to the full 73 5-day steps serie of the validation year 2005. We can observe that the reconstruction closely fits the results provided by the NEMO-PISCES model. The correlation index of the two images in Figure 2 is $97,30 \%$.

We can notice that the discretization induced by the SOM is apparent, yet the general form and intensity are correctly represented, as it becomes clear in Figure 3, where the error graphs tend to have small values.

\section{CONCLUSIONS}

In the present paper we have introduced an inversion method based on SOM and HMM, that is able to reconstruct the vertical profiles of Chlorophyll-A based on satellite images.

The inversion method presented is very efficient in terms of calculations. The method is open ended enough to be applicable for the inversion of the profiles of different bio-geophysical parameters based on satellite imaging. We plan to further validate this method by testing its robustness with satellite imaging and in-situ data, as well as to apply it on different types of profiles, such as oceanic salinity or temperature profiles. A latter goal is to expand the method to take spatial constraints into consideration, and reconstruct $3 \mathrm{D}$ profiles.

\section{ACKNOWLEDGEMENTS}

We would like to thank the "Délégation Generale de l'Armement" for financing this work.

\section{REFERENCES}

S, Haykin 1999. "9. Self-organizing maps". Neural networks - A comprehensive foundation (2nd ed.). Prentice-Hall

L. E. Baum, T. Petrie, G. Soules, and N. Weiss, 1970. "A maximization technique occurring in the statistical analysis of probabilistic functions of Markov chains", Ann. Math. Statist., vol. 41, no. 1, pp. 164-171.

F. Badran, M. Berrada, J. Brajarda M. Crépon,C. Sorror, S. Thiria, J.-P. Hermand M. Meyer, L. Perichob and M. Asch, 2008. "Inversion of satellite ocean colour imagery and geoacoustic characterization of seabed properties: Variational data inversion using a semiautomatic adjoint approach", Journal of Marine Systems, Volume 69, Issues 1-2, Pages 126-136

C. Moulin, A. Kremeur, A. El Moussaoui, C. Ethe, L. Bopp, E. Dombrowsky, E. Greiner, O. Aumont, P. Brasseur, 2008. "Understanding the interannual variability of the oceanic carbon cycle: Results from the coupled biogeochemical-physical global model PISCES-NEMO", American Geophysical Union, Fall Meeting 2008, abstract \#OS31A-1226Journal of Marine Systems, Volume 69, Issues 1-2, Pages 126136

J. Uitz, H. Claustre, A. Morel, S. B. Hooker, 2006 "Vertical distribution of phytoplankton communities in open ocean: An assessment based on surface chlorophyll", Journal of Geophysical Research Vol.111.

Olivier Cappé, Eric Moulines, and Tobias Rydén, 2005. "Inference in Hidden Markov Models." Springer.

Viterbi AJ, 1967. "Error bounds for convolutional codes and an asymptotically optimum decoding algorithm". IEEE Transactions on Information Theory 13 (2): 260-269.

M.S. Ryan and G.R. Nudd. 1993. The Viterbi Algorithm. Technical Report University of Warwick RR-238 